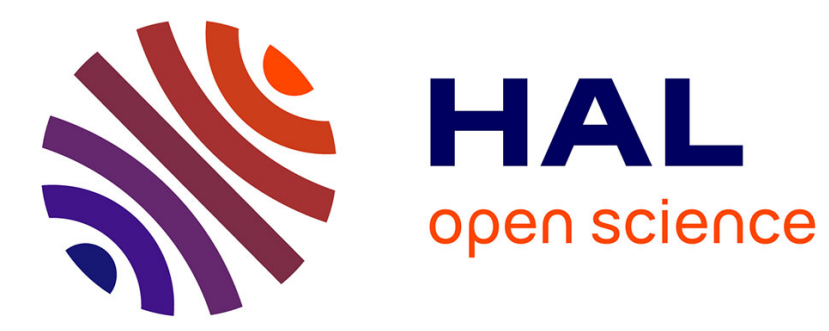

\title{
4DGVF-based filtering of vector-valued images
}

Vincent Jaouen, Paulo Gonzalez, Denis Guilloteau, Irène Buvat, Clovis Tauber

\section{- To cite this version:}

Vincent Jaouen, Paulo Gonzalez, Denis Guilloteau, Irène Buvat, Clovis Tauber. 4DGVF-based filtering of vector-valued images. IEEE International Conference in Image Processing, Oct 2014, Paris, France. pp.1. hal-01064948

\section{HAL Id: hal-01064948 \\ https://hal.science/hal-01064948}

Submitted on 17 Sep 2014

HAL is a multi-disciplinary open access archive for the deposit and dissemination of scientific research documents, whether they are published or not. The documents may come from teaching and research institutions in France or abroad, or from public or private research centers.
L'archive ouverte pluridisciplinaire HAL, est destinée au dépôt et à la diffusion de documents scientifiques de niveau recherche, publiés ou non, émanant des établissements d'enseignement et de recherche français ou étrangers, des laboratoires publics ou privés. 


\title{
4DGVF-BASED FILTERING OF VECTOR-VALUED IMAGES
}

\author{
V. Jaouen ${ }^{1} \quad$ P. Gonzalez G $^{1,2} \quad$ D. Guilloteau ${ }^{1} \quad$ I. Buvat ${ }^{3} \quad$ C. Tauber $^{1}$ \\ ${ }^{1}$ UMR INSERM U930 «Imagerie et cerveau», Université François-Rabelais de Tours \\ 2 bd Tonnelé, 37044 Tours Cedex, France \\ ${ }^{2}$ Universidad Católica del Maule, Talca, Chile \\ ${ }^{3}$ SHFJ/CEA, Orsay, France
}

\begin{abstract}
In this paper, we propose a new method for vector-valued image restoration in order to reduce noise while simultaneously sharpening vector edges. Our approach is a coupled anisotropic diffusion and shock filtering scheme that exploits a new robust $4 \mathrm{DGVF}$ vector field tailored for vector-valued images. The proposed scheme sharpens edges in directions diffused from the entire spatio-spectral information available with a more precise and a more stable sharpening effect along the iterative processing. We validate our method on color images as well as on realistic simulations of dynamic PET images.
\end{abstract}

Index Terms - PDE, Image restoration, Vector-valued images, Gradient vector flow, Positron emission tomography

\section{INTRODUCTION}

Vector-valued images occur in various contexts such as color images, hyperspectral images (e.g. radar, LiDAR), or medical images acquired at different time intervals (e.g. dynamic positron emission tomography (PET) imaging or functional magnetic resonance imaging). Such images require dedicated methods that take profit of additional information provided by the extra dimension available [1]. In particular, edgebased techniques extended to the vector-valued case typically require a redefinition of the notion of gradient. A popular generalization of this notion to vector-valued images or vector gradient, was proposed by Di Zenzo, following considerations from Riemannian geometry [2]. According to Di Zenzo, the image $\mathbf{I}$ is considered as a vector field whose components are the different channels. The gradient direction is then associated with the direction that maximizes a quadratic form of the total differential $d \mathbf{I}$ of $\mathbf{I}$. This idea was later developed using the tensor formalism [3], converting this geometric problem into an algebraic one in which the gradient direction can be associated to the dominant eigenvector of a local structure tensor, or second-moment matrix of the image. The gra-

The research leading to these results has received funding from the European Union's Seventh Framework Programme (FP7/2007-2013) under grant agreement HEALTH-F2-2011-278850 (INMiND). dient magnitude is then generally obtained from a combination of the different eigenvalues of the structure tensor. Over the years, Di Zenzo's gradient and the local structure tensor formalism have been used for different applications such as feature or saliency detection [4, 5], image segmentation [6, 7], edge detection (known as vector edge) [8], and image filtering $[1,9,10,11]$.

For single channel images, many non-linear filtering approaches, such as the anisotropic diffusion filter of Perona and Malik (PM) and its refinements [12, 13, 14] have been proposed in order to reduce noise while preserving edge strength. Alvarez and Mazzora proposed a filtering equation that couples PM anisotropic diffusion and a shock filter term $[15,16]$ in order to further enhance the edge signal and restore sharpness. This term can be seen as an inverse diffusion that restores abrupt discontinuities around edges. However, a direct extension of this latter approach to the vector-valued case, by applying it independently on each channel, is not satisfying. Indeed, edges are often difficult to identify accurately in individual channels of vector-valued images, and independent morphological operations acting in the different channels are likely to create false spectral characteristics, such as false colors [17]. Based on the geometrical considerations of Di Zenzo, Tschumperlé and Deriche extended the Alvarez-Mazorra formulation to the vector-valued case by combining a nonlinear diffusion term, a shock filter term and a data-fidelity term for color image restoration [10]. By acting on all channels in the same fashion, this method reduces incoherent restoration of the vector components. However, shock filters rely on the localization of the Laplacian zerocrossings and therefore remain very sensitive to noise, even when coupled to vector diffusion schemes.

In this paper, we propose a novel approach for vectorvalued image sharpening and denoising based on a coupled vector diffusion and shock filtering scheme. The proposed approach exploits sharpening directions drawn from a new 4D gradient vector flow (4DGVF) field that propagates and regularizes vector gradient information throughout the image. By partially transferring the vector edges localization and orientation tasks the 4DGVF field, we define more accurate and 
robust directions along which blur is compensated in a stable fashion. As all image channels are sharpened in coherent directions, the proposed method also reduces the appearance of spurious spectral characteristics. To a certain extent, this approach can be considered as a generalization of the GVFbased anisotropic diffusion model proposed by $\mathrm{Yu}$ and Chua for 2D grayscale images to the vector-valued case [18]. We validate our method on synthetic color images and realistic simulations of 4D $(3 \mathrm{D}+t)$ dynamic PET images, and compare our results to the approaches of Yu and Chua [18] and of Tschumperlé and Deriche [10].

\section{PROPOSED METHOD}

In this section, we present our vector-valued restoration approach based on the 4DGVF field, a gradient vector flow (GVF) field tailored for vector-valued images [19]. We start by defining a vector geometry in order to characterize vector edges at a local scale, both in amplitude and in direction. These geometrical considerations are the foundation upon which the 4DGVF field and the proposed regularization scheme are built.

\subsection{Definition of a vector geometry}

In the continuous domain, we denote by $\mathbf{I}$ a $n \mathrm{D}$ vectorvalued image constituted of $M$ channels :

$$
\mathbf{I}(\mathbf{x}, c):\left(\Omega_{s} \otimes \Omega_{c}\right) \in \mathbb{R}^{n} \otimes \mathbb{N} \rightarrow \mathbb{R},
$$

where $\Omega_{s}$ is the nD spatial domain of the image and $\Omega_{c}$ the channel dimension. $\mathbf{x}=\left(x_{1}, \ldots, x_{n}\right) \in \Omega_{s}$ is the voxel position in each channel. We denote by $I_{k}$ the $k^{\text {th }}$ channel of the image.

According to Di Zenzo, $\mathbf{I}$ is a $n \mathrm{D} \rightarrow M \mathrm{D}$ vector field. The gradient field of the multichannel image is locally oriented in the direction which maximizes the quadratic form of the total differential $d \mathbf{I}$ of $\mathbf{I}$ [2] :

$$
d \mathbf{I}=\sum_{i=1}^{n} \frac{\partial \mathbf{I}}{\partial x_{i}} d x_{i}
$$

We define a weighted quadratic form for $d \mathbf{I}$, or first fundamental form :

$$
\|d \mathbf{I}\|_{\omega}^{2}=d \mathbf{x}^{T} \mathbf{G}_{\omega} d \mathbf{x},
$$

with $G_{\omega}$ a regularized, weighted structure tensor of the image [20]

$$
G_{\omega}=K_{\sigma} * \sum_{k=1}^{M} \omega_{k}\left(\nabla I_{k} \otimes \nabla I_{k}^{T}\right),
$$

where $K_{\sigma}$ is a Gaussian kernel of scale $\sigma$, $*$ is the convolution operation, $\otimes$ is the tensor product, and $\omega_{k}$ is a weighting factor for channel $I_{k}$. The weighting factors allow to characterize the reliability of the different channels. Without a priori estimation, a natural generalization of Di Zenzo's approach is to weight all channels equally by choosing $\omega_{k}=1 / M, \forall k$.

Let $\lambda_{1} \geq \ldots \geq \lambda_{n}$ be the ordered set of eigenvalues of the structure tensor $G_{\omega}$ and $\left\{\vec{\theta}_{1}, \ldots, \vec{\theta}_{n}\right\}$ the associated orthonormal eigenvector set. The eigenvalues $\lambda_{i}$ give the scalar rates of change of the first fundamental form in a local basis of extremal variations. The dominant eigenvector $\vec{\theta}_{1}$ associated with $\lambda_{1}$ is collinear to the vector gradient, while the remaining eigenvectors span the hyperplane tangent to the local isophote. In order to measure the vector gradient amplitude, several combinations of the eigenvalues can be found in the literature [10]. In our study, we choose a coherence norm proposed by Weickert in [21]:

$$
\|d \mathbf{I}\|_{\omega}^{2}=\sum_{i=1}^{n-1} \sum_{j=i+1}^{m}\left(\lambda_{i}-\lambda_{j}\right)^{2} .
$$

This norm measures the amount of local anisotropy, a generalization to the $n \mathrm{D}$ case of the norm presented by Sapiro in [1]. This measure exhibits oriented gradient patterns in the image. We thus define a measure $N_{\omega}$ of the vector edge amplitude.

$$
N_{\omega}:=\|d \mathbf{I}\|_{\omega} .
$$

\subsection{DGVF field}

We have recently proposed the 4DGVF vector field for the robust segmentation of vector-valued images with active surfaces [22]. This field is obtained through a gradient vector flow-like scheme that propagates vector gradients in the image in a nonlinear fashion [19]. It exploits both amplitude and directional information contained in the analysis of the structure tensor, on the contrary of previous approaches that rely on the norm of the tensor only [23, 24].

We define a vectorial edge map $\overrightarrow{\mathbf{V}}$, a vector field collinear to the local dominant eigenvectors of $G_{\omega}$, but oriented toward the nearest vector edges :

$$
\overrightarrow{\mathbf{V}}=\vec{\theta}_{+} \operatorname{sign}<\vec{\theta}_{+}, \nabla N_{\omega}>.
$$

The 4DGVF field is obtained by nonlinear diffusion of the components of $\overrightarrow{\mathbf{V}}$ throughout the image. It is defined as the steady-state solution $\overrightarrow{\mathbf{F}}_{\text {ext }}$ of the following vector partial differential equation :

$$
\frac{\partial \overrightarrow{\mathbf{F}}_{e x t}}{\partial t}=g\left(N_{\omega}\right) \Delta \overrightarrow{\mathbf{F}}_{e x t}-h\left(N_{\omega}\right)\left(\overrightarrow{\mathbf{F}}_{e x t}-\overrightarrow{\mathbf{V}}\right),
$$

where $g(s)=\mathrm{e}^{-|\nabla s| / \kappa}$ and $h=1-g$ are two functions that control the tradeoff between the first and second terms through parameter $\kappa$ [19], and $\Delta$ is the vector Laplace operator. In the vicinity of vector edges, as measured by $N_{\omega}$, the directions of $\overrightarrow{\mathbf{F}}_{\text {ext }}$ are constrained by $\overrightarrow{\mathbf{V}}$, while isotropic diffusion of $\overrightarrow{\mathbf{F}}_{\text {ext }}$ prevails in homogeneous regions. We obtain a regularized vector field oriented locally toward the nearest vector edge. 


\subsection{DRSF vector regularization}

We propose a new regularization approach for $n \mathrm{D}$ vectorvalued images that combines anisotropic diffusion and 4DGVFregularized shock filtering. The proposed $4 \mathrm{D}$ regularized shock filter (4DRSF) approach is driven by the following set of coupled partial differential equations :

$$
\begin{aligned}
& \frac{\partial I_{k}}{\partial t}=c_{D_{+}}\left(N_{\omega}\right) \frac{\partial^{2} I_{k}}{\partial \vec{\theta}_{1}^{2}}+c_{D_{-}}\left(N_{\omega}\right) \sum_{i=2}^{n} \frac{\partial^{2} I_{k}}{\partial \vec{\theta}_{i}^{2}} \\
& +\alpha\left({ }^{0} I_{k}-I_{k}\right)-c_{S}\left(N_{\omega}\right) \operatorname{sign}\left(<\overrightarrow{\mathbf{F}}, \frac{\nabla I_{k}}{\left|\nabla I_{k}\right|}>\right)\left|\frac{\partial I_{k}}{\partial \overrightarrow{\theta_{1}}}\right|,
\end{aligned}
$$

where operators $\partial / \partial \vec{\theta}_{i}$ and $\partial^{2} / \partial \vec{\theta}_{i}{ }^{2}$ respectively correspond to the directional derivative of first and second order in the $\vec{\theta}_{i}$ direction and where $<,>$ is the dot product.

The first two terms in equation (8) respectively correspond to a diffusion along the vector gradient direction and along the hyperplane tangent to the local isophote, weighted by diffusion coefficients $c_{D_{+}}$and $c_{D_{-}}$, decreasing functions of $N_{\omega}$. The third term is a classical data-attachment term controlled by parameter $\alpha$, where ${ }^{0} I_{k}$ denotes the original $k^{\text {th }}$ channel. The last term in eq. (8) is the 4DGVF-based shock filter term. It sharpens vector edges in robust directions established prior to diffusion by the 4DGVF field $\overrightarrow{\mathbf{F}}_{\text {ext }}$. $c_{S}$ is a decreasing function of $N_{\omega}$ so that sharpening occurs more near edges than in flat regions. While previous shock filter based regularization schemes $([15,10])$ sharpen edges in the direction of the curvature zero-crossings, which may change along the iterations, such a scheme guarantees a stable edge-enhancement behavior along robust directions determined by the 4DGVF field.

\subsection{Implementation}

We implemented the 4DRSF regularization method using MATLAB. We used the following diffusion and shock coefficients : $c_{-}(s)=\frac{1}{\sqrt{1+\frac{s}{r}}}, c_{+}=c_{-}^{2}$ and $c_{S}=1-c_{-}$, where $r$ is a scale parameter. In order to handle data with similar orders of magnitude, all intensities were scaled to the range [0 1$]$.

\section{RESULTS}

We tested our method on two different data sets for which ground truths were available : synthetic color images and realistic simulations of dynamic PET images.

\subsection{Comparative evaluation}

We compared the 4DRSF approach to three other methods : 1) an isotropic Gaussian filter applied in each channel $I_{k}$ independently ; 2) the GVF-based anisotropic diffusion model of Yu and Chua for single-channel images [18]. This method couples anisotropic diffusion with a shock filter term that exploits directions established prior to diffusion by a classical GVF vector field. As for the Gaussian filter, this method was applied in each channel independently ; 3) the regularization approach of Tschumperlé and Deriche, which takes profit of Di Zenzo's vector gradient in order to reduce noise and to sharpen edges in a coherent way along the channels of vectorvalued images [10].

The parameters of all methods were established so as to minimize the root mean square error (RMSE) between the result and the ground truth. We evaluated two quantitative criteria : the RMSE and the signal-to-noise ratio (SNR) [25].

\subsection{Synthetic color images}

We generated a dataset of 50 synthetic $2 \mathrm{D}$ color images having various levels of Gaussian blur and of additive Gaussian noise. The filtering process was performed in the $R G B$ color space. The ground truth is displayed in Fig. 1a and a representative image of the dataset is shown in Fig. 1b. Results of the different approaches are shown in figures 1c-f. For this image, the isotropic Gaussian filter (Fig. 1c) reduced noise at the expense of lowering the spatial resolution. The anisotropic diffusion filter of Yu and Chua (Fig. 1d) reduced noise rather efficiently across the image while providing a sharp rendering of edges. However, a spurious edge sharpening behavior occurred in flat regions. Moreover, false colors were produced around edges. This can be explained by the fact that edges are not sharpened with the same amplitude or along the same directions in the $R, G$ and $B$ channels. The approach of Tschumperlé and Deriche (Fig. 1e) led to a well-rendered image, mostly avoiding the above mentioned issues. However, vector edges were less sharpened that with the method of Yu and Chua, as shocks are developed in varying directions along the iterative treatment, causing the spatial smearing of edges. A visual inspection suggests that the proposed 4DRSF approach was better able to restore the original piecewise constant aspect of the image. These observations were confirmed by the quantitative results displayed in table 1. For this dataset, the Gaussian filter (Gaus.), the approach of $\mathrm{Yu}$ and Chua (Yu.), the approach of Tschumperlé and Deriche (Tsch.) and the proposed 4DRSF approach increased the SNR of the unfiltered image (U.I.) by $42 \%, 63 \%$, $61 \%$ and $65 \%$ respectively.

\subsection{Dynamic PET image simulations}

The validation of filtering results using clinical images is difficult due to lack of ground truth. We assessed the 4DRSF regularization approach with quantitative results on realistic Monte Carlo simulations of dynamic PET images. Three images with different levels of noise were generated using GATE, a highly realistic PET image simulator based on the CERN's GEANT4 particle interaction platform [26]. Noise 


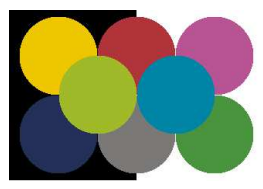

(a)

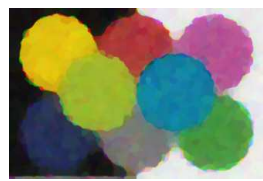

(d)

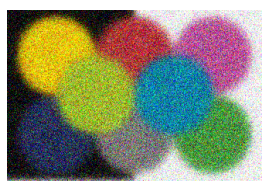

(b)

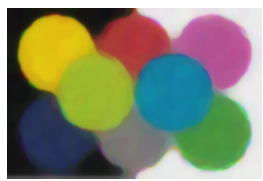

(e)

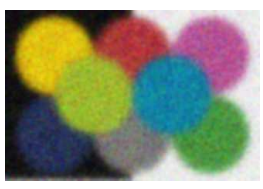

(c)

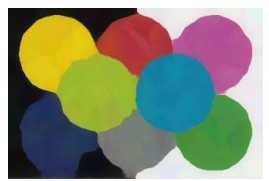

(f)

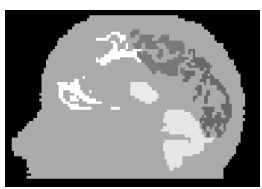

(a)

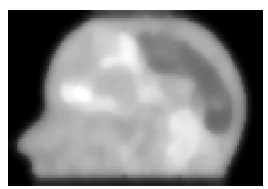

(d)

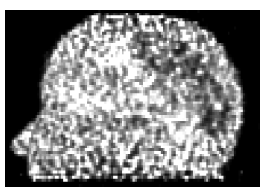

(b)

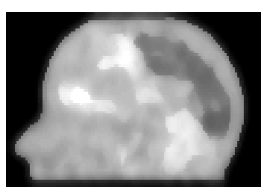

(e)

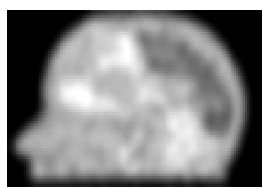

(c)

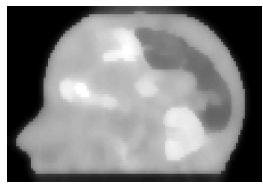

(f)
Fig. 1. Results for the synthetic image. (a) Ground truth, (b) unprocessed image, (c) isotropic Gaussian smoothing, (d) Yu and Chua channel-by-channel approach, (e) Tschumperlé and Deriche approach, (f) proposed 4DRSF approach.

levels varied between these images due to the number of iterations (it) and subsets (s) used by the OP-OSEM reconstruction algorithm (first image : 5it/10s, second image : 2it/16s, third image : 10it/16s.) We used the Zubal head phantom [27], a phantom of the main brain structures, as a voxelized source for the simulated tomographic reconstructions, constituted each of 20 time frames (channels). Results on a sagittal slice of one of the simulations are shown in figure 2, using the same ordering as the one used in figure 1.

While the isotropic Gaussian filter (Fig. 2c) lowered the spatial resolution, the channel-by-channel approach of Yu and Chua (Fig. 2d) led to better denoising. However, it failed at providing sharp boundaries and the image remained blurred. Indeed, this method does not take advantage of the redundancy of the edge signal along the channels in order to lower its sensitivity to noise. The GVF fields calculated in individual channels were too much affected by noise and thus failed at providing relevant sharpening directions. The vector-valued restoration approach of Tschumperlé and Deriche (Fig. 2e) sharpened edges in a coherent way and benefited of the spectral information to identify and to locate edges more precisely. However, the geometry of the different tissues was altered during the process. For example, the thalamus and the frontal lobe regions have been artificially joined due to spurious diffusion behavior. This can be explained by the displacements of the Laplacian zero-crossings along the iterations. Once again, the 4DRSF approach provided both better denoising and better sharpening ability, a property which was confirmed by the quantitative results displayed in the last column of table 1 .

To illustrate the method in a preclinical context, an example on a real [18F]-DPA-714 dynamic PET image is shown in Fig. 3. Quinoleic acid was injected in the right striatum of a rat, producing a hypersignal in the image displayed. The filtering result was consistent with the expected morphology of the pathological region.
Fig. 2. Sagittal slice of a representative channel of a dynamic PET image simulation. (a) Ground truth, (b) unprocessed image, (c) isotropic Gaussian smoothing, (d) Yu and Chua channel-by-channel approach, (e) Tschumperlé and Deriche approach, (f) proposed 4DRSF approach.

Table 1. Average scores for the two datasets

\begin{tabular}{l|c|c|c|c} 
& \multicolumn{2}{|c|}{ 2D color images } & \multicolumn{2}{c}{ 4D PET simulations } \\
& RMSE & SNR $(d B)$ & RMSE & SNR $(d B)$ \\
\hline U.I. & $.15 \pm .04$ & $11.0 \pm 1.9$ & $.27 \pm .09$ & $07.9 \pm 3.6$ \\
Gaus. & $.14 \pm .03$ & $11.5 \pm 1.7$ & $.15 \pm .04$ & $10.7 \pm 1.0$ \\
Yu. & $.11 \pm .01$ & $14.9 \pm 0.3$ & $.12 \pm .01$ & $11.2 \pm 0.4$ \\
Tsch. & $.10 \pm .01$ & $14.9 \pm 0.3$ & $.11 \pm .01$ & $11.7 \pm 0.8$ \\
4DRSF & $\mathbf{. 0 9} \pm . \mathbf{0 1}$ & $\mathbf{1 5 . 2} \pm \mathbf{0 . 3}$ & $\mathbf{. 1 0} \pm . \mathbf{0 1}$ & $\mathbf{1 2 . 9} \pm \mathbf{1 . 9}$
\end{tabular}

\section{CONCLUSION}

We have proposed a new restoration method for vectorvalued images that allows to increase the SNR while simultaneously sharpening vector edges. The proposed approach exploits robust sharpening directions derived from a new vector field that takes profit of the entire spatio-spectral information available in order to better identify both edges strength and direction. Results obtained on synthetic images confirm the potentiality of the proposed method for the restoration of $2 \mathrm{D}$ color and $3 \mathrm{D}+t$ images.
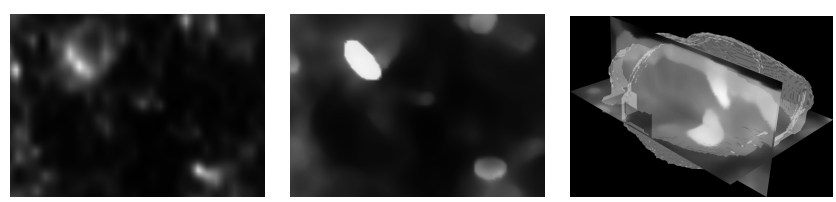

Fig. 3. Real [18F]DPA-714 PET acquisition of a rat brain. left : original image. middle,right : 4DRSF filtering result 


\section{REFERENCES}

[1] G. Sapiro and D.L. Ringach, "Anisotropic diffusion of multivalued images with applications to color filtering," Image Processing, IEEE Transactions on, vol. 5, no. 11, pp. 1582-1586, 1996.

[2] S. Di Zenzo, "A note on the gradient of a multi-image," Computer Vision, Graphics, and Image Processing, vol. 33, no. 1, pp. 116-125, 1986.

[3] H.C. Lee and D.R. Cok, "Detecting boundaries in a vector field," Signal Processing, IEEE Transactions on, vol. 39, no. 5, pp. 1181-1194, 1991.

[4] J. Van De Weijer et al., "Robust photometric invariant features from the color tensor," Image Processing, IEEE Transactions on, vol. 15, no. 1, pp. 118-127, 2006.

[5] G. Piella, "Image fusion for enhanced visualization : A variational approach," International Journal of Computer Vision, vol. 83, no. 1, pp. 1-11, 2009.

[6] G. Sapiro, "Vector (self) snakes : A geometric framework for color, texture, and multiscale image segmentation," in Image Processing, 1996. Proceedings., International Conference on. IEEE, 1996, vol. 1, pp. 817-820.

[7] X. Xie and M. Mirmehdi, "RAGS : Region-aided geometric snake," Image Processing, IEEE Transactions on, vol. 13, no. 5, pp. 640-652, 2004.

[8] A. Cumani, "Edge detection in multispectral images," CVGIP : Graphical models and image processing, vol. 53, no. 1, pp. 40-51, 1991.

[9] T.F. Chan, B.Y. Sandberg, and L. Vese, "Active contours without edges for vector-valued images," Journal of Visual Communication and Image Representation, vol. 11, no. 2, pp. 130-141, 2000.

[10] D. Tschumperlé and R. Deriche, "Diffusion PDEs on vector-valued images," Signal Processing Magazine, IEEE, vol. 19, no. 5, pp. 16-25, 2002.

[11] D. Tschumperlé and R. Deriche, "Vector-valued image regularization with PDEs : A common framework for different applications," Pattern Analysis and Machine Intelligence, IEEE Transactions on, vol. 27, no. 4, pp. 506-517, 2005.

[12] P. Perona and J. Malik, "Scale-space and edge detection using anisotropic diffusion," Pattern Analysis and Machine Intelligence, IEEE Transactions on, vol. 12, no. 7, pp. 629-639, 1990.

[13] F. Catté et al. "Image selective smoothing and edge detection by nonlinear diffusion," SIAM Journal on Numerical analysis, vol. 29, no. 1, pp. 182-193, 1992.
[14] P. Kornprobst et al., "Nonlinear operators in image restoration," in Computer Vision and Pattern Recognition, 1997. Proceedings., 1997 IEEE Computer Society Conference on. IEEE, 1997, pp. 325-330.

[15] L. Alvarez and L. Mazorra, "Signal and image restoration using shock filters and anisotropic diffusion," SIAM Journal on Numerical Analysis, vol. 31, no. 2, pp. 590605, 1994.

[16] S. Osher and L. I. Rudin, "Feature-oriented image enhancement using shock filters," SIAM Journal on $\mathrm{Nu}$ merical Analysis, vol. 27, no. 4, pp. 919-940, 1990.

[17] J. Serra, "The "false colour" problem," in Mathematical Morphology and Its Application to Signal and Image Processing, pp. 13-23. Springer, 2009.

[18] H. Yu and C.S. Chua, "GVF-based anisotropic diffusion models," Image Processing, IEEE Transactions on, vol. 15, no. 6, pp. 1517-1524, 2006.

[19] C. Xu and J.L. Prince, "Generalized gradient vector flow external forces for active contours," Signal Processing, vol. 71, no. 2, pp. 131-139, 1998.

[20] J. Weickert, Anisotropic diffusion in image processing, vol. 1, Teubner Stuttgart, 1998.

[21] J. Weickert, "Coherence-enhancing diffusion of colour images," Image and Vision Computing, vol. 17, no. 3, pp. 201-212, 1999.

[22] V. Jaouen et al. "4-D gradient vector flow : segmentation par surface active pour images multi-composantes," in ORASIS-Journées francophones des jeunes chercheurs en vision par ordinateur, 2013.

[23] L. Yang, P. Meer, and D.J. Foran, "Unsupervised segmentation based on robust estimation and color active contour models," Information Technology in Biomedicine, IEEE Transactions on, vol. 9, no. 3, pp. 475-486, 2005.

[24] V. Jaouen et al. "Vector-based active surfaces for segmentation of dynamic PET images," in Biomedical Imaging (ISBI), 2013 IEEE 10th International Symposium on. IEEE, 2013, pp. 61-64.

[25] W. K. Pratt, Digital Image Processing : PIKS Inside, John Wiley \& Sons, Inc., New York, NY, USA, 3rd edition, 2001.

[26] S. Jan et al., "GATE v6 : a major enhancement of the gate simulation platform enabling modelling of CT and radiotherapy," Physics in medicine and biology, vol. 56, no. 4, pp. 881, 2011.

[27] G. Zubal et al., "Computerized three-dimensional segmented human anatomy," Medical Physics-New YorkInstitute of Physics, vol. 21, no. 2, pp. 299-302, 1994. 